\title{
ACUTE ENCEPHALOPATHY: WHAT TO SUSPECT AND HOW TO PREVENT?
}

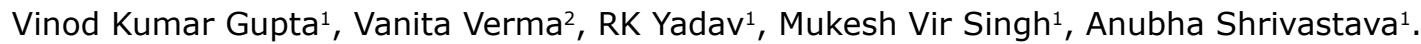 \\ ${ }^{1}$ Department of Pediatrics, MLN Medical College, Prayagraj, Uttar Pradesh, India, \\ ${ }^{2}$ National Diagnostics, New Delhi, India.
}

\section{KEYWORDS}

acute necrotizing encephalopathy, childhood

\section{ARTICLE HISTORY}

Received 12 January 2021

Accepted 20 February 2021

\section{Clinical Problem:}

A 9-year-old girl presented with convulsions followed by unconsciousness. She had fever and cough for one week. On examination, Glasgow coma score (GCS) was $8 / 15$. There were no meningeal signs or focal weakness. Fundus examination was normal. Other systemic examination was normal. Investigations showed hemoglobin $11 \mathrm{~g} / \mathrm{dl}$, total leucocyte count 4200/cumm with $52 \%$ polymorphs and $39 \%$ lymphocytes, serum bilirubin $0.5 \mathrm{mg} / \mathrm{dL}$ with normal transaminases and INR of 1.9. Arterial blood gas showed $\mathrm{pH}$ of 7.38 and bicarbonate $23 \mathrm{mEq} / \mathrm{L}$. Lumbar puncture showed clear cerebrospinal fluid (CSF) with normal pressure, 71.4 $\mathrm{mg} / \mathrm{dL}$ glucose, $16.2 \mathrm{mg} / \mathrm{dL}$ protein, 4 cells/cumm (mostly polymorphs and few degenerated cells) and no bacteria. Nasopharyngeal swab was negative for Covid-19 RT-PCR. Blood and CSF was negative for Japanese Encephalitis IgM. Testing for influenza virus was unavailable at our centre. MRI brain showed symmetrical hyper-intense signal intensity on T2W/ FLAIR, restricted diffusion on DWI and signal drop (apparent diffusion coefficient) involving bilateral ganglio-thalamic complexes, brain stem and cerebral peduncles (Figure 1 ). Injection ceftriaxone $(100 \mathrm{mg} /$ $\mathrm{kg} /$ day in 2 divided doses), vancomycin $(60 \mathrm{mg} / \mathrm{kg} /$ day in 4 divided doses) and acyclovir ( $45 \mathrm{mg} / \mathrm{kg} /$ day in 3 divided doses) were started intravenously (IV). Oseltamivir was unavailable. Convulsions were controlled with levericetam ( $40 \mathrm{mg} / \mathrm{kg} /$ day in 2 divided doses). Intravenous Immunoglobulin (IVIG) $(30 \mathrm{mg} /$ $\mathrm{kg} /$ day for 3 days) followed by oral prednisolone ( 2 $\mathrm{mg} / \mathrm{kg} /$ day in 2 divided doses) were also given. On $3^{\text {rd }}$ day, patient showed improved sensorium and no further convulsions. On follow-up at three months, distal athetoid movements remained but repeat MRI brain showed resolution.

What is the diagnosis?

\section{Discussion:}

Acute necrotising encephalopathy of childhood (ANEC). Mizuguchi et al described ANEC in Japan in 1995. ${ }^{1}$ Most cases are from East Asia but not limited to any particular race. ${ }^{2}$ ANEC follows infection with

Address for Correspondance: $\mathrm{Dr}$. Anubha Shrivastava, 84, Lukerganj, Prayagraj, Uttar Pradesh 211016, India.

Email: anubhashrivastava@rediffmail.com

(C)2021 Pediatric Oncall
Figure 1. MRI brain shows thalamic hyperintensity on $\mathrm{T} 2 \mathrm{~W}$ and restricted diffusion in bilateral thalamus on DWI.

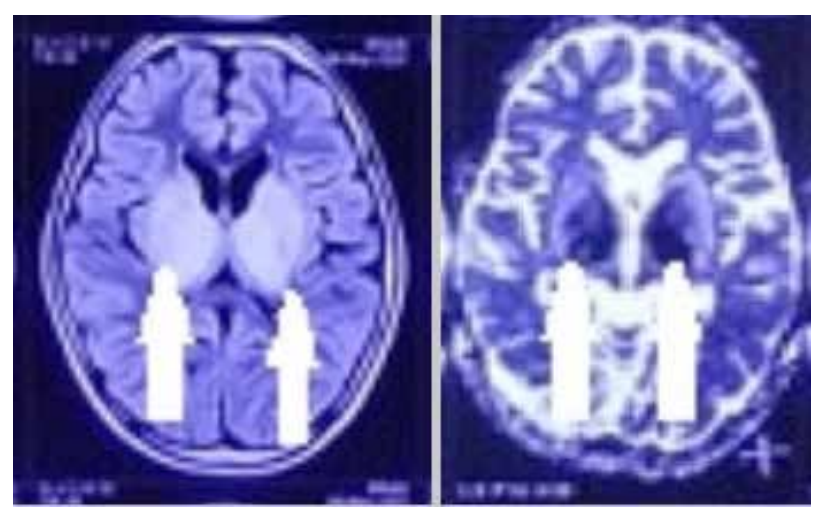

influenza, Herpes Simplex Virus, Human Herpes Virus-6, Parainfluenza virus, Varicella Zoster Virus, reovirus, rotavirus, enterovirus, measles, coxsackie-A9 and mycoplasma. ${ }^{3}$ ANEC occurs within days of fever and presents with convulsions and coma. ${ }^{1}$ Acute manifestations are febrile seizures, movement disorder, frontal lobe encephalopathy and multiorgan failure..$^{2,3}$ Subacute manifestations are Guillain-Barre syndrome, transverse myelitis, acute disseminated encephalomyelitis, cerebellitis and myositis. Late manifestations are parkinsonism and encephalitis lethargica. ${ }^{3}$ Patients of $2009 \mathrm{H} 1 \mathrm{~N} 1$ influenza pandemic showed heightened neurological complications and ANEC compared to 2004-2008 seasonal influenza. ${ }^{3}$ Cytokine storm following viral infection leads to systemic immune response causing multiorgan injury, brain cell apoptosis and cerebral edema from disruption of blood-brain barrier in ANEC. ${ }^{2,3}$ RANBP2 genetic polymorphism is associated with recurrent episodes of ANEC with viral respiratory infections. ${ }^{4}$ Central necrosis, surrounding cytotoxic oedema and peripheral vasogenic oedema forms pathological basis for MRI appearance. ${ }^{3}$ T1W show hypointense lesions, T2W/FLAIR display corresponding mixed hyperintense signals with restricted diffusion on DWI and circular enhancement on contrast MRI. ${ }^{2}$ Normal CSF and characteristic bilateral thalamic involvement with diffusion restriction help in making diagnosis. ${ }^{5}$ Acute disseminated encephalomyelitis, neurovascular accidents, Reye's syndrome, mitochondrial dysfunction and fulminant hepatitis are other differentials. ${ }^{3,6}$ Outcomes range 
from full recovery $(<10 \%)$, neurologic sequelae in survivors to fulminant progression in majority. ${ }^{3} \mathrm{Li}$ et al observed positive correlation between MRI findings and clinical outcome. ${ }^{2}$ Observation of hemorrhage and local tissue loss on MRI predicts a poor prognosis. ${ }^{2}$ Selective vulnerability of thalami seems to be determining factor. ${ }^{6}$ Antivirals, immunoglobulin, plasmapheresis, antithrombin-III, and therapeutic hypothermia has been tried. ${ }^{3}$ High dose steroids used in earlier stages determines the prognosis. ${ }^{6}$ Follow-up imaging shows regression of lesions with residual cortical atrophy, cystic changes and haemosiderin deposition. Functional recovery following rehabilitation has been reported. ${ }^{3}$ Given the potential for ANEC recurrence, monitoring of children with history of neurological complications following respiratory illness is indicated during influenza season with prompt testing and antiviral therapy. ${ }^{4}$ Annual influenza vaccination is important for ANEC survivors and their household contacts. ${ }^{4}$

\section{Compliance with ethical standards}

Funding: None

Conflict of Interest: None

\section{References:}

1. Mizuguchi M, Abe J, Mikkaichi K, Noma S, Yoshida K, Yamanaka T, Kamoshita S. Acute necrotising encephalopathy of childhood: a new syndrome presenting with multifocal, symmetric brain lesions. J Neurol Neurosurg Psychiatry. 1995; 58:555-561.

2. Li H, Sun C, Chi S, Wang Y, Wu L, Qin X. Use of MRI in the diagnosis and prognosis of acute necrotizing encephalopathy in a Chinese teenager. Medicine 2019;98:44.

3. Yoganathan S, Sudhakar SV, James EJ, Thomas MM. Acute necrotising encephalopathy in a child with H1N1 influenza infection: a clinicoradiological diagnosis and follow-up. BMJ Case Rep. 2016 Jan 11;2016:bcr2015213429.

4. Howard A, Uyeki TM, Fergie J. Influenza-Associated Acute Necrotizing Encephalopathy in Siblings. J Pediatric Infect Dis Soc. 2018; 7: e172-177.

5. Sharma M, Sood D, Chauhan NS, Negi P. Acute necrotizing encephalopathy of childhood. Neurol India. 2019;67:610-611.

6. Seo HE, Hwang SK, Choe BH, Cho MH, Park SP, Kwon S. Clinical spectrum and prognostic factors of acute necrotizing encephalopathy in children. J Korean Med Sci. 2010;25:449-453. 